\title{
Les Indes Orientales au carrefour des Empires (1760-1830)
}

Bernard Gainot et Massimiliano Vaghi

\section{Q OpenEdition \\ 1 Journals}

Édition électronique

URL : https://journals.openedition.org/ahrf/13043

DOI : $10.4000 /$ ahrf.13043

ISSN : 1952-403X

Éditeur :

Armand Colin, Société des études robespierristes

Édition imprimée

Date de publication : 1 mars 2014

Pagination : 3-8

ISBN : 978-2-200-9083-2790-8

ISSN : 0003-4436

Référence électronique

Bernard Gainot et Massimiliano Vaghi, «Les Indes Orientales au carrefour des Empires (1760-1830) », Annales historiques de la Révolution française [En ligne], 375 I janvier-mars 2014, mis en ligne le 05 juillet 2021, consulté le 05 juillet 2021. URL : http://journals.openedition.org/ahrf/13043 ; DOI : https:// doi.org/10.4000/ahrf.13043 
INTRODUCTION

\section{LES INDES ORIENTALES AU CARREFOUR DES EMPIRES (1760-1830)}

Bernard GAINOT et Massimiliano VAGHI

Les méthodes et les perspectives de recherches autour de l'histoire globale suscitent actuellement l'intérêt et les interrogations du monde universitaire français, comme en témoigne la très récente rubrique de notre revue sur ce thème ${ }^{1}$, mais elles se trouvent encore fort peu différenciées de l'histoire atlantique. Nous avons donc souhaité, dans ce numéro spécial, mettre l'accent sur les Indes orientales à l'âge des révolutions européennes, les mondes asiatiques ouverts aux Européens, à l'exception de la Chine des Qing et du Japon des Tokugawa qui, dans la période considérée (1760 1830) sont des sociétés fermées.

Les contacts directs entre l'Europe et les Indes Orientales, après une longue période d'extranéité substantielle pendant le Moyen Âge, reprennent vigueur par la découverte portugaise de la route du Cap au début du XVI ${ }^{\mathrm{e}}$ siècle. L'espace géopolitique de l'Océan Indien commence, ainsi, à susciter de plus en plus l'intérêt économique des puissances européennes et, en même temps, à stimuler l'imagination des hommes cultivés, tant religieux que laïques.

Si l'Europe a repris la route des Indes Orientales après un long oubli, il ne faut pas oublier que cet espace était déjà très actif, riche et vivant - tant du point de vue économique que culturel - depuis quelques millénaires : en effet, comme toutes les études actuelles le montrent, l'Océan Indien était un carrefour des Empires bien avant l'arrivée des Européens ${ }^{2}$, c'est-à-dire

(1) Rubrique « Regards croisés ; la Révolution française à l'heure du gobal turn » sous la direction de Karine RANCE, avec des contributions de Paul CHENEY, Allan Forest, Lynn HunT et Mathias MIDDELL, dans $A H R F$, n ${ }^{\circ}$ 4, 2013, p. 157 - 185.

(2) Voir, par exemple, l'étude pionnière de Kirti N. CHAUDHURI, Asia before Europe. Economy and Civilisation of the Indian Ocean from the Rise of Islam to 1750, Cambridge, Cambridge University Press, 1990. Voir aussi: Kenneth MCPHERSON, The Indian Ocean : A History of People and the Sea, 
une zone géopolitique ouverte aux voyages et aux trafics, où les marchands et les missionnaires venus d'Europe étaient seulement une des nombreuses présences qui se concurrençaient ${ }^{3}$. Silvia Pizzetti, dans une mise au point historiographique, retrace les singularités de ces espaces emboîtés, tant du point de vue de leurs héritages, commerciaux, culturels, politiques, que de leurs interconnexions croissantes - que la notion d'Empire ne peut encore saisir que de manière imparfaite - et elle souligne l'importance de l'œuvre d'Immanuel Wallerstein à cet égard, tout en insistant sur les recherches très fécondes dans le monde anglo-saxon, autour du concept et des méthodes de la thalassologie.

En particulier, le XVIII ${ }^{\mathrm{e}}$ siècle est caractérisé, à l'échelle globale, comme le dernier siècle pour lequel le «système mondial »n'est pas entièrement soumis à l'Europe, ni du point de vue économique, ni du point de vue de la politique. On peut le considérer, donc, comme la dernière période de l'Âge Moderne. Si, au début du siècle, une nouvelle expansion européenne en Inde - d'abord économique et ensuite politique - va lentement commencer, ayant comme protagonistes principaux les compagnies commerciales de France et d'Angleterre, toutefois cette expansion ne fut ni facile, ni rapide. En effet, c'est seulement à partir de la moitié du siècle que l'Europe, lentement et grâce à sa suprématie technologique et militaire, commença à soumettre et à exploiter économiquement le subcontinent.

D'autre part, évidemment, dans le cas particulier du «théâtre » indien, il faut considérer que ne s'y produisaient pas seulement les acteurs européens : dans le subcontinent, au début du XVIII ${ }^{\mathrm{e}}$ siècle, une fois disparu le dernier des grands empereurs mogols (Aurangzeb, mort en 1707), les potentats locaux se rendirent de facto indépendants de Delhi, même s'ils continuaient formellement de respecter l'autorité nominale du Grand Mogol : l'empire, donc, se transforma d'une monarchie plutôt centralisée en un ensemble de provinces fort autonomes. À cette époque,

London-New Delhi, Oxford University Press, 1998; Michael Naylor PEARSON, The Indian Ocean, London-New York, Routledge, 2003 ; Roy TIRTHANKAR, India in the World Economy : From Antiquity to the Present, Cambridge, Cambridge University Press, 2012 ; et le récent ouvrage de Philippe BeAujard, Les mondes de l'Océan Indien, 2 tomes, Paris, Colin, 2012 (en particulier le tome II, « L'Océan Indien au cœur des globalisations de l'Ancien Monde, VII ${ }^{\mathrm{e}}-\mathrm{XV}^{\mathrm{e}}$ siècles »).

(3) Voir, notamment Michael Naylor PEARSON (dir.), Spices in the Indian Ocean World, Aldershot, Variorum, 1996 ; Ashin DAS GUPTA, Merchants of Maritime India (1500-1800), Aldershot, Variorum, 1994 ; Om PRAKASH (dir.), European Commercial Expansion in Early Modern Asia, Aldershot, Variorum, 1997 ; Pius MaLeKANDATHIL, Maritime India. Trade, Religion and Policy in the Indian Ocean, New Delhi, Primus Books, 2010 ; Abdul SHERIFF, Dhow Cultures of the Indian Ocean. Cosmopolitanism, Commerce and Islam, London, C. Hurst \& Co., 2010 ; et Haneda MASASHI (dir.), Asian Port Cities, 1600-1800. Local and Foreign Cultural Interactions, Singapore-Kyoto, Nus Press-Kyoto University Press, 2009. 
pour les nawabs, tous les Européens actifs en Inde étaient peu importants, tant sur le plan politique que militaire.

Toutefois, malgré cette situation d'instabilité qui compliqua beaucoup les relations entre les officiers des compagnies européennes et les Indiens, l'intérêt des puissances maritimes pour l'Inde augmenta considérablement pendant le XVIII ${ }^{\mathrm{e}}$ siècle, s'étendant peu à peu - à partir de l'Inde à toute la zone géopolitique de l'Océan Indien.

La Péninsule Indienne occupe ainsi une place privilégiée dans ce numéro spécial des Annales Historiques de la Révolution Française. C'est tout d'abord par les récits des voyageurs que la civilisation indienne s'installe dans l'imaginaire du public lettré français. Florence D'Souza présente la place occupée par les récits des voyageurs européens en Inde dans l'Histoire générale des voyages de l'abbé Prévost, compilation monumentale qui couvre toute la seconde moitié du XVIII ${ }^{\mathrm{e}}$ siècle ; place primordiale, en ce sens que les récits des aventuriers portugais en Inde ouvrent la série, comme elle le sera également dans l'autre grande somme d'histoire de la colonisation européenne, L'Histoire des Deux Indes de l'abbé Raynal. Ces récits de voyage alimentent le débat public sur la colonisation, dans la mesure où ils n'occultent pas les violences intrinsèquement liées aux échanges, servent de matériau pour l'émergence de l'anthropologie historique en Europe, nourrissent la vogue de l'orientalisme. Massimiliano Vaghi étudie précisément la place de l'Inde au sein de ce vaste courant orientaliste, passant d'une curiosité empreinte de merveilleux et de pittoresque à une approche plus scientifique vers la fin du XVIII ${ }^{\mathrm{e}}$ siècle. Deux figures importantes se distinguent pour cette approche anthropologique, Anquetil-Duperron et le père jésuite Cœurdoux, avant que l'expertise scientifique ne soit placée sous le signe exclusif de la philologie, au début du XIX $^{\mathrm{e}}$ siècle. Raphaël Malangin fait le tableau de la crise profonde des comptoirs français subsistant après le traité de 1763 ; crise avant tout financière qui rend hypothétique toute tentative de redressement durable de la France dans cette partie du monde. Pourtant, le succès croissant de l'orientalisme, les plans chimériques échafaudés par des entrepreneurs militaires dispersés dans le sous-continent, entretiennent l'illusion d'un contrepoint à l'impérialisme britannique, comme le démontre l'épisode de Tipû Sâhib, le sultan du Mysore, entre 1782 et 1799. C'est à la recherche d'un nouveau Tipû que s'emploie le capitaine général des établissements français des Indes orientales, Charles Decaen, sous l'Empire napoléonien. Bernard Gainot retrace l'univers politique, commercial et idéologique d'un de ses agents secrets établi à Chandernagor, Élzear Morénas. L'engagement contre la seule forme de domination impérialiste de l'époque, la domination 
britannique, se double chez lui d'une véritable empathie pour la civilisation indienne, avant que ses engagements anti-esclavagistes ne l'empêchent de devenir un spécialiste officiel du sanskrit sous la Restauration.

L'observatoire des Français en Asie du sud-est est alors Port-Louis, la ville principale de l' ̂̂le de France, actuelle Île Maurice. Les Mascareignes sont bien placées pour occuper l'intermédiation entre l'Afrique et l'Asie, entre l'économie esclavagiste de plantation et l'économie mercantiliste des comptoirs. Bruno Maillard s'attache au sort d'un groupe d'esclaves de Bourbon (Île de la Réunion) considérés comme dangereux, et déportés à ce titre par les autorités de l'île vers le comptoir de Sainte-Marie, au nord-est de Madagascar, qui fait ainsi figure de colonie pénitentiaire. On voit par là que le retour vers les sociétés africaines d'origine peut prendre un tout autre aspect que celui de la « colonisation libre », à travers laquelle on cherchait un nouveau modèle de travail et d'échanges. La colonie pénitentiaire de Madagascar est une illustration des peines de relégation et de déportation, par lesquelles l' appareil judiciaire colonial cherchait également à surmonter la contradiction entre puissance régalienne et prérogative domaniale.

Port-Louis est également le port de relâche des croisières lointaines vers les mers australes, mais également vers le Pacifique, comme la croisière de Nicolas Baudin (mars 1801-août 1803). L'historiographie a beaucoup mis en exergue l'opposition entre le jeune médecin François Péron, et Nicolas Baudin, mort en 1803, dépositaire des Instructions encore imprégnées de l'anthropologie universaliste des Lumières. Jean-Luc Chappey fait le point sur cette rupture, en apportant une réponse nuancée : l'usage symbolique du dynamomètre pour mesurer la force musculaire des populations sauvages permettant ainsi de séparer ces dernières des populations civilisées, puis de hiérarchiser ces peuples sauvages, ne résume pas à lui seul le bilan de l'expédition dressé par Péron en 1807. Il invite à découvrir les nombreuses observations, et donc les représentations des « sauvages de la mer Pacifique » dans cette époque de transition.

À l'orient des Indes orientales, mais également à l'origine de la présence européenne en Asie, un autre univers se révèle, celui de l'Insulinde, l'archipel indonésien. Angelie Sens propose de replacer celuici au sein d'une thalassologie emblématique, mais en totale recomposition, la thalassologie hollandaise, dans la perspective des recherches historiques actuelles sur l'histoire globale, la nouvelle histoire des Empires, et la période de transition induite par la République batave de 1795, puis le rattachement de l'outre-mer hollandais à l'ensemble hétéroclite français en 1806. Elle souligne l'originalité de la période 1815-1830, période de projets, 
de réformes, dans la perspective d'une colonisation nouvelle, contractuelle. Autour de 1830, cette perspective expérimentale s'évanouit.

À travers les contributions des auteurs de ce numéro - à côté des intérêts politiques et économiques des puissances européennes aux Indes Orientales - on peut noter que la prolifération d'images et d'idées sur l'altérité asiatique, considérées comme une réponse à une situation nouvelle, imprévisible et quelquefois incompréhensible, est en général liée à une époque de bouleversements, peut-être de révolutions tant politiques que culturelles et cognitives, qui, d'une certaine façon, obligent les Européens à prendre position, car la nouveauté ne peut pas laisser indifférents les hommes, en contribuant à créer aussi bien une nouvelle perception des autres, qu'une manière différente de se mettre en relation avec eux.

$\mathrm{Au} \mathrm{XVIII}{ }^{\mathrm{e}}$ siècle les relations entre Europe et Asie deviennent de plus en plus unidirectionnelles : l'Européen connaît et conquiert l'espace asiatique, et le rend sujet aux lois et aux canons occidentaux (esthétiques, historiques, religieux, économiques, politiques, etc.). Toutefois, dans un monde complexe comme celui de l'Asie et de l'Océan Indien, devenu encore plus complexe après l'arrivée des puissances venues d'Europe, l'Européen se définit et se redéfinit grâce au rapport avec l'Autre asiatique.

Jusqu'à aujourd'hui, l'historiographie a considéré l'analyse de l'image et de l'idée de l'Autre comme un point de vue particulier pour essayer de reconstruire l'histoire de la culture européenne, c'est-à-dire pour établir le rôle de la perception et de l'image de l'Autre dans le processus historique de la naissance de l'image de l'Européen, de sa vision du monde et de ses coutumes politiques et morales. En effet, on peut lire l'histoire de la civilisation européenne comme l'histoire d'une succession de « rencontres », comme une « découverte » continuelle (souvent étroitement liée à une sorte d'assujettissement ou de conquête) finalisée - quoique de manière imparfaite et intermittente - à la connaissance et à la compréhension de l'Autre. Les catégories de la rencontre, de la connaissance, de la comparaison, de la curiosité, de la nouveauté se révèlent donc essentielles pour comprendre l'Européen qui, pour cette raison - et malgré sa soidisant supériorité - n'est jamais réellement autosuffisant : il s'agit pour l'Européen d'une auto identification qui naît par une différentiation, et par une comparaison, avec l'Autre, et finalement il peut se reconnaître seulement dans un rapport à sa diversité.

La période comprise entre la fin du XVIII ${ }^{\mathrm{e}}$ et le début du XIX ${ }^{\mathrm{e}}$ siècles se caractérise par d'importantes nouveautés à propos de la construction et 
de l'imaginaire de l'identité des peuples ${ }^{4}$. S'il est vrai que l'Européen reste encore un «producteur » majeur d'images - la naissance du nationalisme et des États nationaux, la reconfiguration des rapports entre les pays d'Europe et ses colonies, l'industrialisation croissante et la « révolution » des transports et la mobilité des hommes garantissent une dimension globale à l'Europe -, par ailleurs l'Autre asiatique est définitivement reconnu comme faisant partie du même temps et de la même "histoire » que l'Europe. Les civilisations orientales restent donc, aux yeux des Européens, fort différentes de celles d'Europe mais elles deviennent de plus en plus reconnaissables et de quelque façon intégrées dans le « monde » européen (non seulement en raison de la colonisation mais aussi grâce à la diffusion des études orientalistes et au succès croissant dans le public des ouvrages sur les Orients).

Les Indes Orientales, en particulier, à partir de la fin du XVIII ${ }^{\mathrm{e}}$ siècle, se détachent peu à peu comme un sous-ensemble autonome à l'intérieur des représentations de l'Orient en général - qui a servi à l'Europe aussi bien de miroir que de repoussoir, en sollicitant une identification ou, au contraire, une démarcation -, devenant différentes d'une manière évidente, pour l'Européen, tant du Proche-Orient que de la Chine et du Japon, grâce à la quantité et à la complexité des religions et des civilisations existantes dans cette zone géopolitique vaste et multiculturelle.

Bernard GAINOT

Massimiliano VAGHI

(4) Sur la pénétration des Lumières en Inde, par exemple, voir Rita SIL, «Images de la Révolution française dans la colonisation et la décolonisation en Inde », dans Michel VovELLE (dir.), L'image de la Révolution française : communications présentées lors du Congrès mondial pour le bicentenaire de la Révolution, Sorbonne, Paris, 6-12 juillet 1989, 4 volumes, Oxford-Paris-New York, Pergamon Press, 1989, vol. III, p. 1812-1822 ; et Kapil RAJ, «L'orientalisme en Inde au tournant du XIX ${ }^{\mathrm{e}}$ siècle: la réponse du mondialisme britannique à l'universalisme de la Révolution française », $A H R F, \mathrm{n}^{\circ} .320,2000$, p. 89-99 (on line, http://ahrf.revues.org/document155.html). 